\title{
Evaluation of the lateral ventricle using MRI in normal micropigs
}

\author{
Mihyun Choi, Namsoon Lee, Kangjae Yi, Junyoung Kim, Mincheol Choi* \\ Department of Veterinary Medical Imaging and Research Institute for Veterinary Science, Seoul National University, \\ Seoul 151-741, Korea \\ (Received: June 13, 2011; Revised: August 01, 2011)
}

\begin{abstract}
This study was undertaken to assess the lateral ventricle, which was some portion of brain and related to congenital anomalies, from 1, 2, 4, and 8 months of age in healthy micropigs. They were induced general anesthesia and performed magnetic resonance imaging (MRI) with a 0.3 Tesla magnet. Each age group was evaluated by three subjects such as lateral ventricular volume, ventricular volume ratio and asymmetry. T1 weighted transverse images were acquired for calculation of lateral ventricular and corresponding brain parenchyma areas. The ratio of bilateral ventricle areas used to analyze the asymmetry. The mean ventricular volumes of each month were $676.74 \pm 25.58 \mathrm{~mm}^{3}$ (1 month-old), 630.64 $\pm 143.84 \mathrm{~mm}^{3}$ (2 month-old), $992.12 \pm 106.03 \mathrm{~mm}^{3}$ (4 month-old) and $1172.62 \pm 237.57 \mathrm{~mm}^{3}$ (8 monthold), respectively. The ventricular volume ratio was the smallest at 2 month-old and re-increased from that age. The ratio was significantly different between 2 month-old and other age groups $(p<0.05)$. The value of bilateral area ratio showed within 1.5 in all experimental animals. Consequently the lateral ventricle showed a positive correlation with aging and symmetric shapes in both sides. The developmental pattern of the lateral ventricle provides basic data in micropigs as an experimental animal model for physiological and neurosurgical approach.
\end{abstract}

Keywords : brain, lateral ventricle, micropigs, MRI

\section{Introduction}

Magnetic resonance imaging (MRI) provides important information about the brain and it has been used to evaluate central nervous system (CNS) diseases of animals [7, 17].

Micropigs are steadily gaining importance as animal models in the field of neurologic research, including dementia, stroke, and congenital anomalies and so on [8, 10]. The micropigs brain, which is gyrencephalic, resembles the human brain more in anatomy, growth and development than do the brains of commonly used small laboratory animals [9]. Important practical aspects of the use of the animals are objective data for comparing normal and disease status. To date, there is few study of the pig brain with MRI. The purpose of this study is to assess the micropigs ventricle from 1 to 8 months of age with MRI. Especially, the onset of ventricular expansion, ventricular symmetry and the developmental change of ventricular volumes and ratios were evaluated.

\section{Materials and Methods}

\begin{abstract}
Animals
Sixteen healthy micropigs at 1,2, 4 and 8 months of age were used in this study. All animals (PWG micropig, PWG Genetics Korea, Korea) were raised under strict SPF barrier system and microbiologically well defined conditions. The micropigs were individually housed indoors in cages, fed dry pig food, and provided with water ad libitum. This study adhered to the strict to the guide line of the 'Guide for the Care and Use of Laboratory Animals' of Seoul National University, Korea.
\end{abstract}

\section{Methods}

MR images of the brain were acquired under general anesthesia. MR images were obtained with a 0.3 Tesla magnet (Airis Vento; Hitachi Company, Japan). Micropigs were placed in dorsal recumbency on the scanning table. $\mathrm{T} 1$ weighted images were acquired in transverse, sagittal

\footnotetext{
*Corresponding author

Tel: +82-2-880-1278, Fax: +82-2-880-8662

E-mail: mcchoi@snu.ac.kr
} 

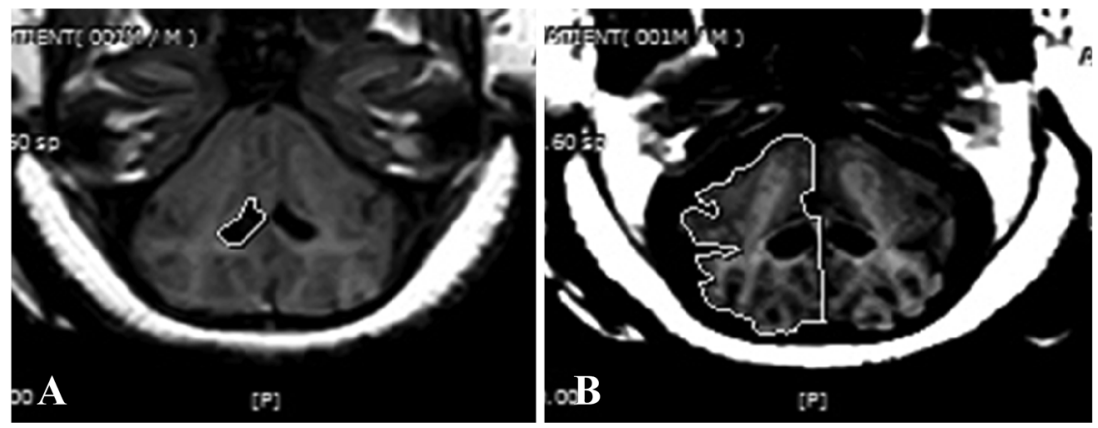

Fig. 1. The areas of the hemisphere (A) and corresponding lateral ventricle (B) were manually calculated on T1 weighted transverse images.

A
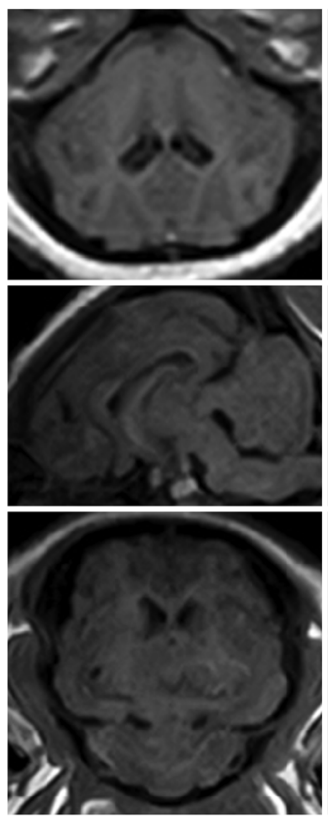

B
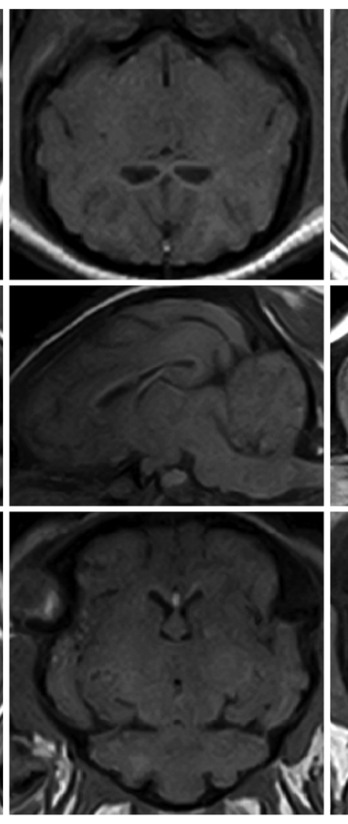

C
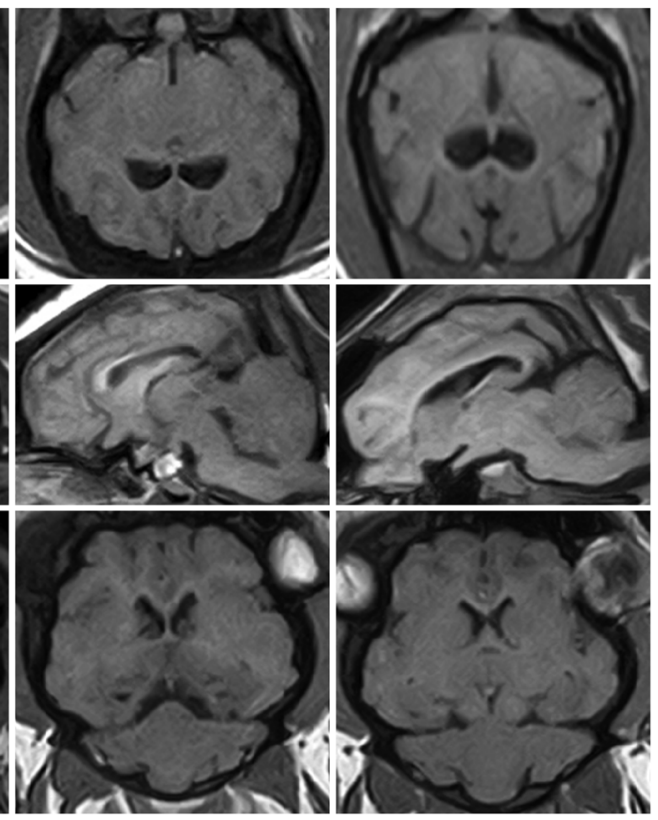

Fig. 2. $T 1$ weighted axial images of three planes at each month-aged. 1 month (A), 2 month (B), 4 month (C) and 8 month (D) age old in micropigs.

and coronal plane using repetition time (TR) of $410 \mathrm{~ms}$ and an echo delay time (TE) of $23 \mathrm{~ms}$. Slice thickness for the all planes was $1.5 \sim 2 \mathrm{~mm}$.

Each month-aged group was evaluated by three values as lateral ventricular volume, ventricular volume ratio and asymmetry. T1 weighted transverse images were acquired for calculation of lateral ventricular and corresponding brain parenchyma areas (Fig. 1). Lateral ventricular volume was the sum of areas on each transverse plane multiplied by slice thickness and volume ratio was calculated by dividing the ventricular areas by brain parenchyma. The ratio of bilateral ventricular areas used to analyze the symmetry, dividing the volume of the right side by that of the left lateral ventricle. In addition, degree of asymmetry was arbitrarily categorized on the basis of the ratio as normal to mild (value $<0.5$ ), moderate $(1.5<$ value $<2.0$ ), or severe (value $>2.0$ ). The images were measured by two observers.

Statistical analysis was performed using SPSS Significant differences among each month-aged groups were determined with independent $t$-tests and $p$-values of $<0.05$ were considered statistically significant. Interobserver agreement was assessed with the kappa statistic with values closest to 1 indicating perfect agreement. 
Table 1. Quantitative ventricular volume analysis

\begin{tabular}{lccccc}
\hline \hline Minipig & LLV & RLV & Total LV & RLV/LLV & BPV \\
\hline 1 month & $318.86 \pm 18.88$ & $357.88 \pm 6.70$ & $676.74 \pm 25.58$ & $1.13 \pm 0.05$ & $5920.58 \pm 590.16$ \\
2 month & $319.38 \pm 78.92$ & $311.26 \pm 65.02$ & $630.64 \pm 143.84$ & $0.98 \pm 0.04$ & $20067.28 \pm 2403.28$ \\
4 month & $490.97 \pm 39.88$ & $501.13 \pm 68.09$ & $992.12 \pm 106.03$ & $1.02 \pm 0.07$ & $21141.27 \pm 1135.12$ \\
8 month & $593.15 \pm 112.33$ & $579.46 \pm 129.86$ & $1172.62 \pm 237.57$ & $0.98 \pm 0.09$ & $23290.16 \pm 1581.56$ \\
\hline
\end{tabular}

LLV: Left lateral ventricular volume, RLV: Right lateral ventricular volume, LV: Lateral ventricular volume, BPV: Brain parenchymal volume.

All data represent mean $\pm \mathrm{SD}(\mathrm{mL})$.

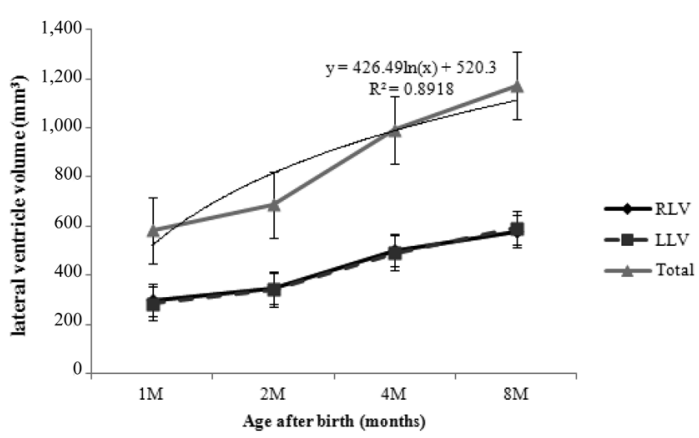

Fig. 3. Changes of the lateral ventricular volume at 1, 2, 4 and 8 month age.

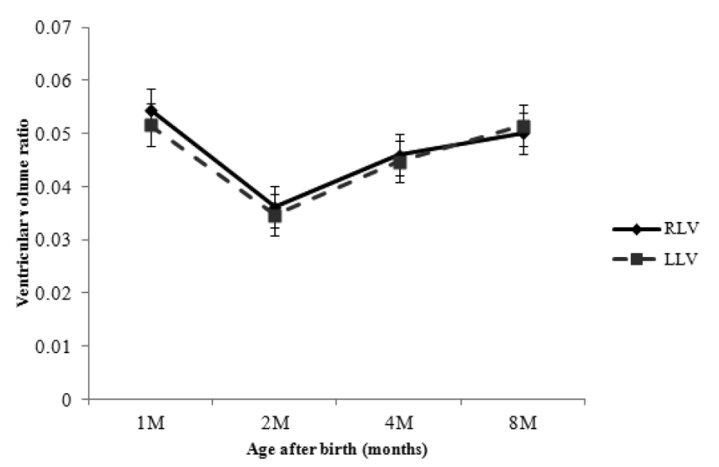

Fig. 4. Changes of the ventricular volume ratio at 1, 2, 4 and 8 month age.

\section{Results}

As the micropigs grow, the size of the lateral ventricles was increased (Figs. 2 and 3). The mean ventricular volumes of each month were $676.74 \pm 25.58$ $\mathrm{mm}^{3}$ (1 month-old), $630.64 \pm 143.84 \mathrm{~mm}^{3}$ ( 2 month-old), $992.12 \pm 106.03 \mathrm{~mm}^{3}$ (4 month-old) and $1172.62 \pm 237.57$ $\mathrm{mm}^{3}$ (8 month-old), respectively. The value of bilateral area ratio showed within 1.5 in all experimental animals (Table 1).
The ventricular volume ratio was the smallest at 2 month-of-age and re-increased from that age. The ratio was significantly different between 2 month-old and other age groups ( $p<0.05$, Fig. 3 ). The value of bilateral area ratios showed within 1.5 in all experimental animals. The inter-observer reproducibility was excellent for all measured images $(k>0.9)$.

\section{Discussion}

MR images provide exact assessment of the size of the lateral ventricles and brain images [1, 2]. Especially, as the enlargement of the lateral ventricles is closely related to neurological disease such as hydrocephalus in clinics, some studies have been reported about this [11, $14,15,17]$. But in the micropigs, to date this study was not well established. But Watanabe et al. [16] demonstrate that the volume of MR-based lateral ventricles in young adult male Gottingen micropigs show about 740 830 $\mathrm{mm}^{3}$, which is similar volume in 4 months of age or over that age group in our experiment. This difference seems to be due to genetic characteristics in the micropigs.

Kii et al. [5] reported that in Beagle-type dogs, the expansion of lateral ventricles were first detectable at 3 4 weeks-old. In this experiment, the lateral ventricles were visible from the 1 month of age, and have been increase as age advanced. In human infants, ventricular size gradually increase up to 6 months of age [13] and it has been reported that by the end of the 1 st week of life a rapid increase in ventricular size occurred [12]. Micropigs also show that the size of the lateral ventricle increase up to 8 months of age, which is to be related to an increase in volume of CSF because of a change from fetal low pressure to the neonatal high pressure circulatory state in human [12]. Gonzalez-Soriano et al. [4] have shown that the enlargement which takes place in the ventricular system with age, which is probably 
related to a general age-related atrophy of neural tissue using dog brain. But in our experiment in which young animals was used, this finding was not revealed and it remains further study.

About the size of the lateral ventricle size and symmetry, Kii et al. [6] and Vullo et al. [15] reported that clinically insignificant ventricular enlargement and asymmetry was common in this group of Beagle dog. These findings are also found in the Labrador Retriever dogs [2].

In the different canine breeds such as Yorkshire Terrier and German Shepherd dogs, relative ventricle area ([ventricle area/ hemisphere area] $\times 100$ ) shows different values (5.3 vs. 1.7) [3]. But we have found that the symmetry of the lateral ventricles in the micropigs was not apparent and the ventricular volume ratio was within 1.17 during the experimental period.

In our experiment, we have found that at 2 months old, the ventricular volume ratio shows the smallest value. This may be caused by the rapid increase of brain size compare to the ventricle size at this time.

\section{Conclusions}

MR imaging is a noninvasive imaging technique to assess morphometric analysis of the ventricular system in micropigs. In normal cases, lateral ventricle showed a positive correlation with aging and symmetric shapes in both sides. The change of ventricular ratio showed ' $\mathrm{V}$ ' shaped graph, which inferred growth of brain parenchyma is faster than lateral ventricle especially until 2 month-old. The developmental pattern of the lateral ventricle provides basic data in micropigs as an experimental animal model for physiological and neurosurgical approach.

\section{Acknowledgments}

This study was supported by a grant (2007 0401034006) from the Biogreen 21 Program, Rural Development Administration, Korea.

\section{References}

1. Assheuer J, Sager M. MRI and CT Atlas of the Dog. 1st ed. pp. 347-403, Blackwell, Berlin, 1997.

2. De Haan CE, Kraft SL, Gavin PR, Wendling LR, Griebenow ML. Normal variation in size of the lateral ventricles of the Labrador Retriever dog as assessed by magnetic resonance imaging. Vet Radiol Ultrasound 1994, 35, 83-86.

3. Esteve-Ratsch B, Kneissl S, Gabler C. Comparative evaluation of the ventricles in the Yorkshire Terrier and the German shepherd dog using low-field MRI. Vet Radiol Ultrasound 2001, 42, 410-413.

4. González-Soriano J, Marín García P, ContrerasRodríguez J, Martínez-Sainz P, Rodríguez-Veiga E. Age-related changes in the ventricular system of the dog brain. Ann Anat 2001, 183, 283-291.

5. Kii S, Uzuka Y, Taura Y, Nakaichi M, Inokuma H, Onishi T. Developmental change of lateral ventricular volume and ratio in Beagle-type dogs up to 7 months of age. Vet Radiol Ultrasound 1998, 39, 185-189.

6. Kii S, Uzuka Y, Taura Y, Nakaichi M, Takeuchi A, Inokuma H, Onishi T. Magnetic resonance imaging of the lateral ventricles in Beagle-type dogs. Vet Radiol Ultrasound 1997, 38, 430-433.

7. Kimotsuki T, Nagaoka T, Yasuda M, Tamahara S, Matsuki N, Ono K. Changes of magnetic resonance imaging on the brain in beagle dogs with aging. $\mathrm{J}$ Vet Med Sci 2005, 67, 961-967.

8. Kuzmuk KN, Schook LB. Pigs as a model for biomedical sciences. In: Rothschild MF, Ruvinsky A (eds.). The Genetics of the Pig. 2nd ed. pp. 426-444, CAB international, Wallingford, 2011.

9. Lind NM, Moustgaard A, Jelsing J, Vajta G, Cumming P, Hansen AK. The use of pigs in neuroscience: modeling brain disorders. Neurosci Biobehav Rev 2007, 31, 728-751.

10. Mikkelsen M, Møller A, Jensen LH, Pedersen A, Harajehi JB, Pakkenberg H. MPTP-induced Parkinsonism in minipigs: a behavioral, biochemical, and histological study. Neurotoxicol Teratol 1999, 21, 169-175.

11. Nam JW, Choi CB, Woo DC, Ryu KN, Kang EH, Chang HS, Lee DW, Choe BY, Kim HY. Evaluation of hydrocephalic ventricular alterations in Maltese dogs using low field MRI. Int J Appl Res Vet Med 2011, 9, 58-67.

12. Saliba E, Bertrand P, Gold F, Vaillant MC, Laugier J. Area of lateral ventricles measured on cranial ultrasonography in preterm infants: reference range. Arch Dis Child 1990, 65, 1029-1032.

13. Shah PS, Sarvaiya JB, Rawal JR, Kabra SK, Patel VB, Joshi RN. Normal ventricular size and ventriculo- 
hemispheric ratio in infants upto 6 months of age by cranial ultrasonography. Indian Pediatr 1992, 29, 439442.

14. Spaulding KA, Sharp NJH. Ultrasonographic imaging of the lateral cerebral ventricles in the dog. Vet Radiol 1990, 31, 59-64.

15. Vullo T, Korenman E, Manzo RP, Gomez DG, Deck MDF, Cahill PT. Diagnosis of cerebral ventriculomegaly in normal adult Beagles using quantitative MRI. Vet Radiol Ultrasound 1997, 38, 277-281.
16. Watanabe H, Andersen F, Simonsen CZ, Evans SM, Gjedde A, Cumming P; DaNeX study group. MRbased statistical atlas of the Göttingen minipig brain. Neuroimage 2001, 14, 1089-1096.

17. Woo DC, Choi CB, Nam JW, Ryu KN, Jahng GH, Lee SH, Lee DW, Kim SY, Kim HY, Ahn KJ, Choe BY. Quantitative analysis of hydrocephalic ventricular alterations in Yorkshire terriers using magnetic resonance imaging. Vet Med (Praha) 2010, 55, 125-132. 